COMMENT. Rasmussen's encephalitis is characterized by intractable seizures of focal onset, usually an epilepsia partialis continua, and chronic inflammation with progressive hemispheric neurologic deterioration. In the final residual, atrophic stage of the disorder, seizures decrease in frequency and the neurologic deficit becomes static. The differentiation between a viral cause or an autoimmune mechanism remains to be determined.

\title{
PROSPECTIVE DIAGNOSIS AND TREATMENT OF PANDAS
}

Twelve school-aged children with new-onset pediatric autoimmune neuropsychiatric disorder with group A streptococcal infection (PANDAS) were identified over a 3-year period (1998-2000) at the University of Rochester, NY. An acute onset of severe obsessive-compulsive disorder was associated with an acute group-A beta-hemolytic streptococcal (GABHS) pharyngitis, confirmed by positive throat culture and/or rapid antigen detection, and in 3 cases, very high antideoxyribonuclease antibody titers. All patients were treated with penicillin, amoxicillin or a cephalosporin for 10 days, and eradication of GABHS was followed by immediate resolution of the OCD symptoms. Recurrence of OCD in 6 patients was associated with acute GABHS that responded to antibiotic therapy. The age at onset of OCD was 5-12 years, predominantly in boys (1.4:1), and the initial OCD episode occurred from September through April, the peak seasonal occurrence of GABHS. Symptoms of OCD, meeting DSMIV diagnostic criteria, included compulsive hand washing or excessive toilet hygiene, compulsive daytime urinary urgency without infection, and age-inappropriate separation anxiety when leaving their mothers. Four children (33\%) had neurological disorders in addition to OCD, including recurrent tics, transient $\mathrm{ADHD}$, or choreiform movements. This is the first prospective study to confirm the PANDAS syndrome and its response to treatment with penicillin. (Murphy ML, Pichichero ME. Prospective identification and treatment of children with pediatric autoimmune neuropsychiatric disorder associated with group A streptococcal; infection (PANDAS). Arch Pediatr Adolesc Med April 2002;156Ú356-361). (Reprints: Michael E Pichichero MD, Elmwood Pediatric Group, University of Rochester Medical Center, 601 Elmwood Avenue, Box 672, Rochester, NY).

COMMENT. This study corroborates the association between streptococcal infection and the acute onset of neuropsychiatric symptoms. It also demonstrates the benefit of treatment with penicillin for the tonsillitis or pharyngitis with concomitant recovery from the OCD, tics and sometimes ADHD symptomatology. A possible diagnosis of PANDAS should be considered in new cases of OCD or tics, especially when symptoms are acute in onset.

\section{SEIZURE DISORDERS}

\section{SCN1A GENE MUTATIONS IN SEVERE INFANTILE MYOCLONIC EPILEPSY}

Ten novel mutations of SCN1A were found in in a pair of monozygotic twins and 12 unrelated Japanese infants with severe myoclonic epilepsy in infancy (SMEI) examined at the Brain Science Institute, Saitama; and National Epilepsy Center, Shizuoko, Japan. Of the 10 heterozygous mutations, 3 were frameshift and 7 nonsense mutations. These rather than missense mutations are the major causes of SMEI. Missense mutations of the same gene result in generalized epilepsy with febrile sezures plus (GEFS plus). (Sugawara T, Mazaki-Miyazaki E, Fukushima K et al. Neurology April (1 of 2) 2002;58:1122-1124). (Reprints: Dr K Yamakawa, Laboratory 